\title{
Preparation of human serum albumin nanoparticles using a chemometric technique
}

\author{
Nastaran Hosseinifar ${ }^{1}$ Amir Abdolah Mehrdad Sharif ${ }^{1,4} \cdot$ Navid Goodarzi $^{2}$. \\ Mohsen Amini ${ }^{3} \cdot$ Rassoul Dinarvand ${ }^{2}$
}

Received: 23 June 2017 / Accepted: 5 October 2017 / Published online: 30 November 2017

(C) The Author(s) 2017. This article is an open access publication

\begin{abstract}
Human serum albumin (HSA), a versatile protein carrier for drug delivery, is an ideal material to fabricate nanoparticles for drug delivery systems. These nanoparticles can accumulate in tumor interstitium due to the enhanced permeability and retention effect. The most important characteristics of HSA nanoparticles are particle size, shape, and zeta potential. A chemometric approach was applied for HSA nanoparticles' size optimization in this study. The effects of three experimental parameters; pressure $(P)$ or power, organic solvent volume $(V)$, and time $(T)$, were investigated under sonication and high-pressure homogenization, using multivariate analysis. The trials were performed based on the Box-Behnken experimental design. The criteria for the appraisal of the descriptive ability of a multinomial were $R^{2}=0.819$, standard error $=20.420$, and $F$-ratio $=19.550$. The method was optimized with respect to the nanoparticles' size as a response. The Box-Behnken experimental design was applied to optimize and trial the robustness of the HSA nanoparticle preparation method.
\end{abstract}

Keywords Nanoparticles · Human serum albumin · Drug delivery · Chemometrics · Experimental design · Optimization

\section{Introduction}

The non-specific distribution of cytotoxic drug molecules and adverse effects are the major drawbacks of conventional chemotherapy methods. Nanoparticulate drug delivery systems have been extensively investigated as a robust strategy in targeted drug delivery in the field of pharmaceuticals [1-3]. Human serum albumin (HSA) has been shown to be biodegradable, biocompatible, and nonimmunogenic, and it has been used extensively as a protein

Amir Abdolah Mehrdad Sharif

a_sharif@iau-tnb.ac.ir

1 Department of Applied Chemistry, Faculty of Chemistry, Islamic Azad University, Tehran-North Branch, Tehran, Iran

2 Nanotechnology Research Centre, Faculty of Pharmacy, Tehran University of Medical Sciences, Tehran, Iran

3 Department of Medicinal Chemistry, Faculty of Pharmacy, Tehran University of Medical Sciences, Tehran, Iran

4 Department of Analytical Chemistry, Faculty of Chemistry, Islamic Azad University, North Tehran Branch, Tehran, Iran carrier. HSA may protect drug molecules from degradation, increase water solubility, increase drug absorption by facilitating diffusion through epithelium, change the drug tissue distribution profile and pharmacokinetic, and/or improve intracellular penetration and distribution [4-10]. Albumin-bound nanoparticles can facilitate drug accumulation in tumors by a well-known passive targeting mechanism called the enhanced permeability and retention (EPR) effect. In the EPR effect, the blood vessel walls in a tumor are dilated, leaky or defective, and the endothelium is disorganized with nano-sized fenestrations [11-14]. Nanocarriers, ranging in size from 20 to $250 \mathrm{~nm}$, can extravasate fluid from the vessels inside the interstitial space. Endothelial pores, ranging in size from 10 to $1000 \mathrm{~nm}$, are suitable for passing nanoparticles. Moreover, the lymphatic vessels have impaired function in a tumor, which contributes to inefficient drainage of fluids from the tumor tissue. Because nanocarriers that enter the tumor are not removed efficiently, they accumulate $[15,16]$. Albumin nanoparticles bind to albumin-binding glycoprotein (gp60) with a high affinity in the nanomolar concentrations and 
inducing gp60 clustering and transcytosis. Both gp60bound and fluid phase albumin are carried through the tumor endothelium subsequently releasing nanoparticles into the tumor tissue. After nanoparticle entrance to the tumor interstitium, the accumulation of albumin in tumor cells is possibly facilitated by secreted protein, acidic and rich in cysteine (SPARC), which is a matricellular, collagen-binding protein. SPARC receptors on the surface of cells may facilitate cellular uptake of HSA and release drug conjugates [14, 17, 18].

Nanoparticle albumin-bound $\left(\mathrm{nab}^{\mathrm{TM}}\right)$ technology is the commercial process used to encapsulate lipophilic drugs into HSA nanoparticles [19, 20]. In this method, lipophilic drugs are dissolved in an organic solvent and mixed with an aqueous solution of HSA. This emulsion is then passed through a HPH to reduce the particle size to $100-200 \mathrm{~nm}$ $[20,21]$.

Optimization refers to improve the function of a system, a process, or a product to achieve the maximum yield. Experimental design and optimization are tools for systemically examining different types of problems that arise in research, development, and production. Therefore, after defining the variables and responses of experiments, they can be planned and performed in such a way that maximum information is gained from a minimum number of experiments [22].

Optimizing nanoparticles' preparation is a complex process due to the wide range of parameters involved in controlling their size. Therefore, a set of experiments was designed and conducted using a Box-Behnken design. Moreover, the results were evaluated to determine if a quantitative relationship existed between the variables. Recently, chemometrics have been utilized in analytical optimization methods because they provide more advantages such as less laboratory work. In addition, the chemometric approach has led to the development of models that allow researchers to evaluate the correlation and statistical significance of the parameters in a study and assess the interacting factors [23].

In the present study, HSA nanoparticles were produced using a method inspired from nab technology under sonication and homogenization. First, sonication was used, and then the experiments continued with HPH. The results were optimized for each matrix. Finally, a model was created with the aim of identifying the impact of various factors on the size and polydispersity index (PdI) of the HSA nanoparticles in order to predict the best condition for fabricating the smallest size and the lowest PdI value.

\section{Materials and methods}

\section{Materials}

Potassium dihydrogen phosphate $\left(\mathrm{KH}_{2} \mathrm{PO}_{4}\right)$ was purchased from Merck (Darmstadt, Germany), Acetonitrile was purchased from Duksan (Ansan, South Korea), paclitaxel was purchased from Xingcheng Chemphar Co. (Zhejiang, China), and HSA was supplied from Sigma Aldrich (Hamburg, Germany). Ultra-purified water was used throughout the analysis, and all the other chemicals were of analytical grade.

\section{Methods}

\section{Experimental design}

Before applying the multiple regression technique, it is necessary to choose an experimental design approach. This design will define which experiments should be carried under the study conditions. For this purpose, the present study used two experimental methods: sonication and HPH (Tables 1 and 2, respectively). These parameters were chosen because their most significant effect on the size and PdI of nanoparticles. The levels were selected based on the initial experiments' results. Moreover, a three-factor, at three levels, Box-Behnken experimental design was used to test the robustness of the method three times. The middle values experiment provided a more precise estimate of the adequacy of the model. All statistical analysis was performed on range-scaled factor values of $[-1,+1]$.

Table 1 The three factors and the corresponding three level settings under sonication

\begin{tabular}{llll}
\hline Level & Power (amplitude) & Time $(\mathrm{min})$ & Volume $(\mathrm{mL})$ \\
\hline-1 & 20 & 2 & 0.5 \\
0 & 40 & 6 & 1 \\
+1 & 60 & 9 & 1.5 \\
\hline
\end{tabular}

Table 2 The three factors and the corresponding three level settings under $\mathrm{HPH}$

\begin{tabular}{lccl}
\hline Level & Pressure $($ bar $)$ & Time $(\mathrm{min})$ & Volume $(\mathrm{mL})$ \\
\hline-1 & 700 & 2 & 0.5 \\
0 & 1000 & 6 & 2 \\
+1 & 1500 & 10 & 4 \\
\hline
\end{tabular}




\section{Statistical methods}

The coefficients of the model were calculated using a multiple regression technique and were validated using ANOVA. The independent sample $t$ test is used for comparison of DLS size of HSA nanoparticles under sonication and $\mathrm{HPH}$.

\section{Preparation of drug-loaded human serum albumin nanoparticles}

The HSA nanoparticles were prepared using a method inspired by nab technology. Paclitaxel as chemotherapeutic drug (27 mg) was dissolved in various amounts of acetonitrile. HSA (243 mg) was mixed with $100 \mathrm{~mL}$ of

Organic phase
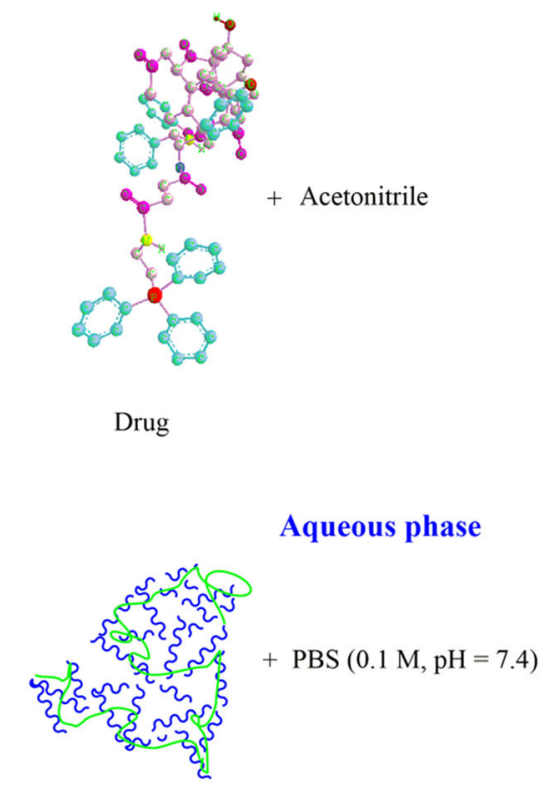

Human Serum Albumin

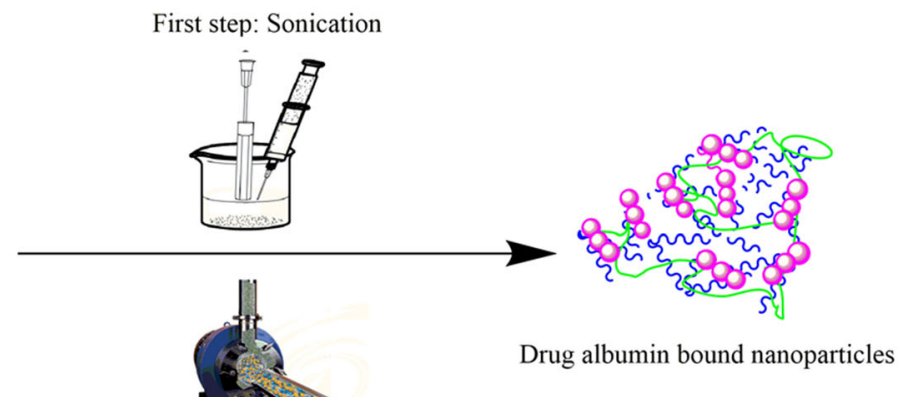

Second step: HPH

Fig. 1 HSA drug albumin-bound nanoparticles preparation

Table 3 Experimental conditions for Box-Behnken design and average response for particle size and PdI with sonicator

\begin{tabular}{lllllrl}
\hline Trial & Power (amplitude) & Time $(\mathrm{min})$ & Volume $(\mathrm{mL})$ & Size $(\mathrm{nm})$ & SD $(n=3)$ & PdI \\
\hline 1 & - & - & 0 & 165 & 5.000 & 0.226 \\
2 & + & - & 0 & 209 & 6.244 & 0.226 \\
3 & - & + & 0 & 166 & 4.041 & 0.306 \\
4 & + & + & 0 & 188 & 6.429 & 0.299 \\
5 & - & 0 & - & 222 & 16.165 & 0.312 \\
6 & + & 0 & - & 187 & 2.309 & 0.278 \\
7 & - & 0 & + & 331 & 52.003 & 0.278 \\
8 & + & 0 & + & 211 & 11.503 & 0.319 \\
9 & 0 & - & - & 186 & 12.701 & 0.202 \\
10 & 0 & + & - & 197 & 10.785 & 0.311 \\
11 & 0 & - & + & 204 & 8.621 & 0.321 \\
12 & 0 & + & + & 175 & 12.701 & 0.342 \\
13 & 0 & 0 & 0 & 155 & 8.736 & 0.349 \\
14 & 0 & 0 & 0 & 130 & 10.148 & 0.333 \\
15 & 0 & 0 & 0 & 151 & 2.081 & 0.355 \\
16 & 0 & 0 & 0 & 149 & 17.776 & 0.324 \\
17 & 0 & 0 & 0 & 236 & 18.583 & 0.337 \\
\hline
\end{tabular}


phosphate-buffered saline (PBS) $(0.1 \mathrm{M}, \mathrm{pH}=7.4)$. The drug in its organic phase was loaded into the HSA solution under sonication (Probe Sonicator, QSONICA Q700, New York City, USA), as shown in Fig. 1. In the following, the mixture was homogenized using a high-pressure homogenizer (HPH 2000/4-SH5; IKA, Staufen, Germany) to form drug albumin nanoparticles in the size range of 100-200 $\mathrm{nm}$. The remaining acetonitrile was evaporated under mild vacuum conditions for $30 \mathrm{~min}$ using a rotary evaporator. The mixture was lyophilized with Christ (ALPHA 2-4 LD plus Osterode am Harz, Germany) to achieve a homogenous white cake.
Particle size, size distribution, zeta potential, and surface morphology

The size, size distribution, and zeta potential of the HSA nanoparticles were determined after sonication and after HPH with the dynamic light scattering (DLS) method using a Malvern Zetasizer NanoZS (Malvern Instruments, Worcestershire, UK). In addition, the shape and surface morphology of the HSA nanoparticles were characterized after sonication and after HPH using a scanning electron microscope (SEM) (KYKY-EM3200, KYKY Technology, Shanghai, China). In addition, the particles' resolution was
Table 4 Experimental conditions for Box-Behnken design and average response for particle size and PdI under HPH

\begin{tabular}{lllllcl}
\hline Trial & Pressure (bar) & Time $(\mathrm{min})$ & Volume $(\mathrm{mL})$ & Size $(\mathrm{nm})$ & SD $(n=3)$ & PdI \\
\hline 1 & - & 0 & - & 250 & 6.027 & 0.408 \\
2 & - & 0 & + & 287 & 6.027 & 0.518 \\
3 & + & 0 & - & 193 & 7.211 & 0.291 \\
4 & + & 0 & + & 204 & 10.58 & 0.392 \\
5 & 0 & - & - & 212 & 5.773 & 0.268 \\
6 & 0 & - & + & 234 & 12.055 & 0.337 \\
7 & 0 & + & - & 152 & 5.131 & 0.194 \\
8 & 0 & + & + & 213 & 4.041 & 0.434 \\
9 & - & - & 0 & 306 & 31.214 & 0.315 \\
10 & + & - & - & 210 & 14.224 & 0.342 \\
11 & - & + & 0 & 161 & 13.203 & 0.415 \\
12 & + & + & - & 130 & 11.239 & 0.175 \\
13 & 0 & 0 & 0 & 209 & 11.060 & 0.262 \\
14 & 0 & 0 & 0 & 211 & 7.371 & 0.365 \\
15 & 0 & 0 & 0 & 219 & 16.258 & 0.333 \\
16 & 0 & 0 & 0 & 214 & 9.848 & 0.386 \\
17 & 0 & 0 & 0 & 222 & 13.650 & 0.298 \\
\hline
\end{tabular}

A

$\begin{array}{rlllll} & & \text { Diam. }(\mathbf{n m}) & \% \text { Intensity } & \text { Width (nm) } \\ \text { Z-Average (d.nm): } & 165 & \text { Peak 1: } & 212 & 100.0 & 96.1 \\ \text { Pdl: }: & 0.226 & \text { Peak 2: } & 0.00 & 0.0 & 0.00 \\ \text { Intercept: } & 0.899 & \text { Peak 3: } & 0.00 & 0.0 & 0.00\end{array}$

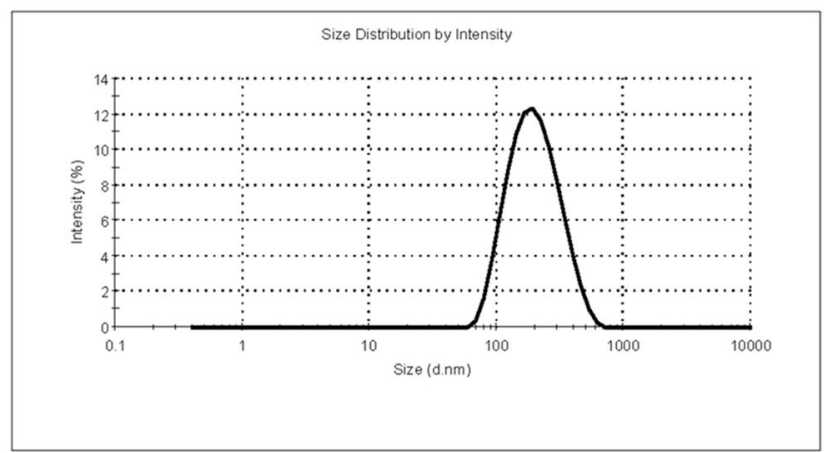

\section{B}

$\begin{array}{rlllll} & & & \text { Diam. }(\mathbf{n m}) & \text { \% Intensity } & \text { Width }(\mathrm{nm}) \\ \text { Z-Average (d.nm): } & 152 & \text { Peak 1: } & 182 & 100.0 & 81.9 \\ \text { Pdl: } & 0.194 & \text { Peak 2: } & 0.00 & 0.0 & 0.00 \\ \text { Intercept: } & 0.923 & \text { Peak 3: } & 0.00 & 0.0 & 0.00\end{array}$

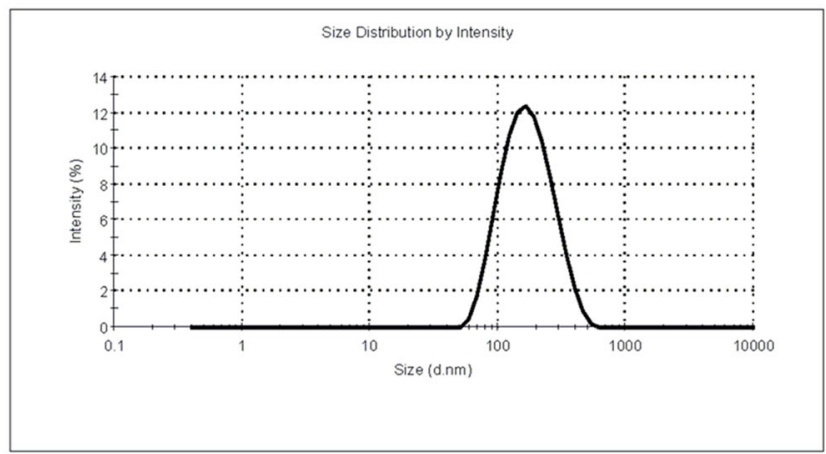

Fig. 2 Size distribution of nanoparticles preparation under sonication (a) and HPH (b) which shows that the particle diameters of both methods could be adjusted between 100 and $200 \mathrm{~nm}$ 

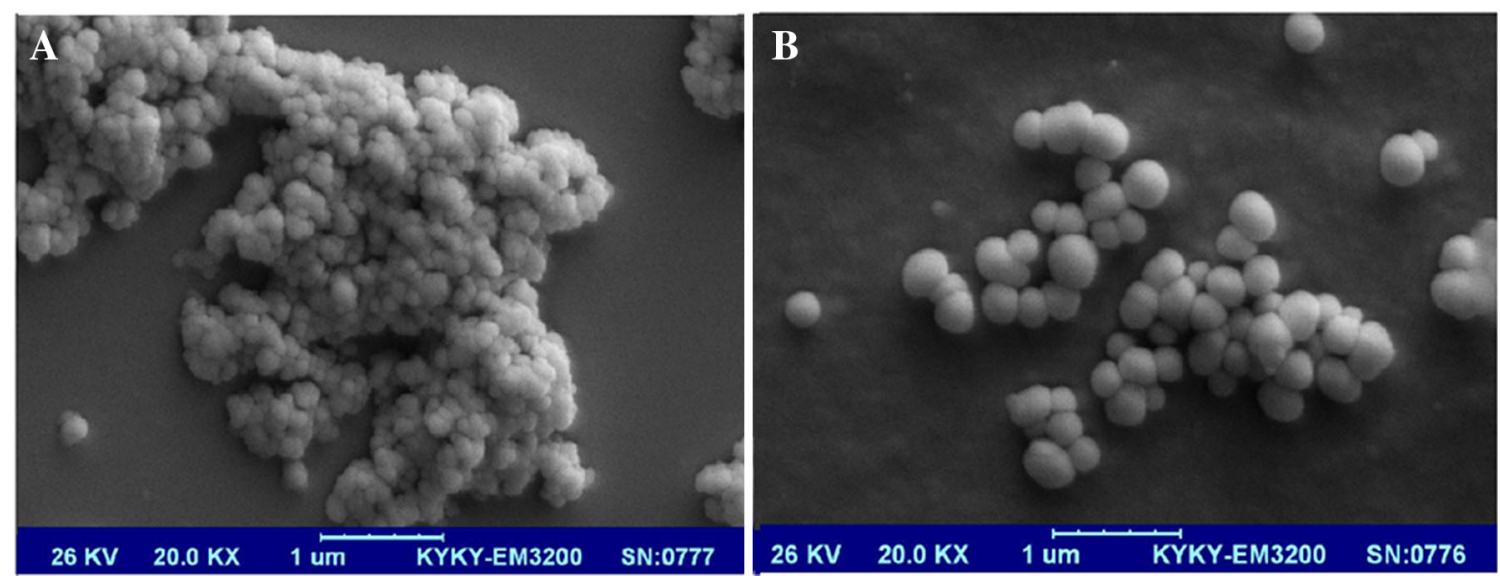

Fig. 3 SEM images of the nanoparticles made using sonication (a) and HPH (b). The sonic sample was aggregate but HPH sample was stable

Fig. 4 TEM images of the nanoparticles made using sonication (a, b) and HPH (c, d). The sonic samples were aggregate but HPH samples were stable
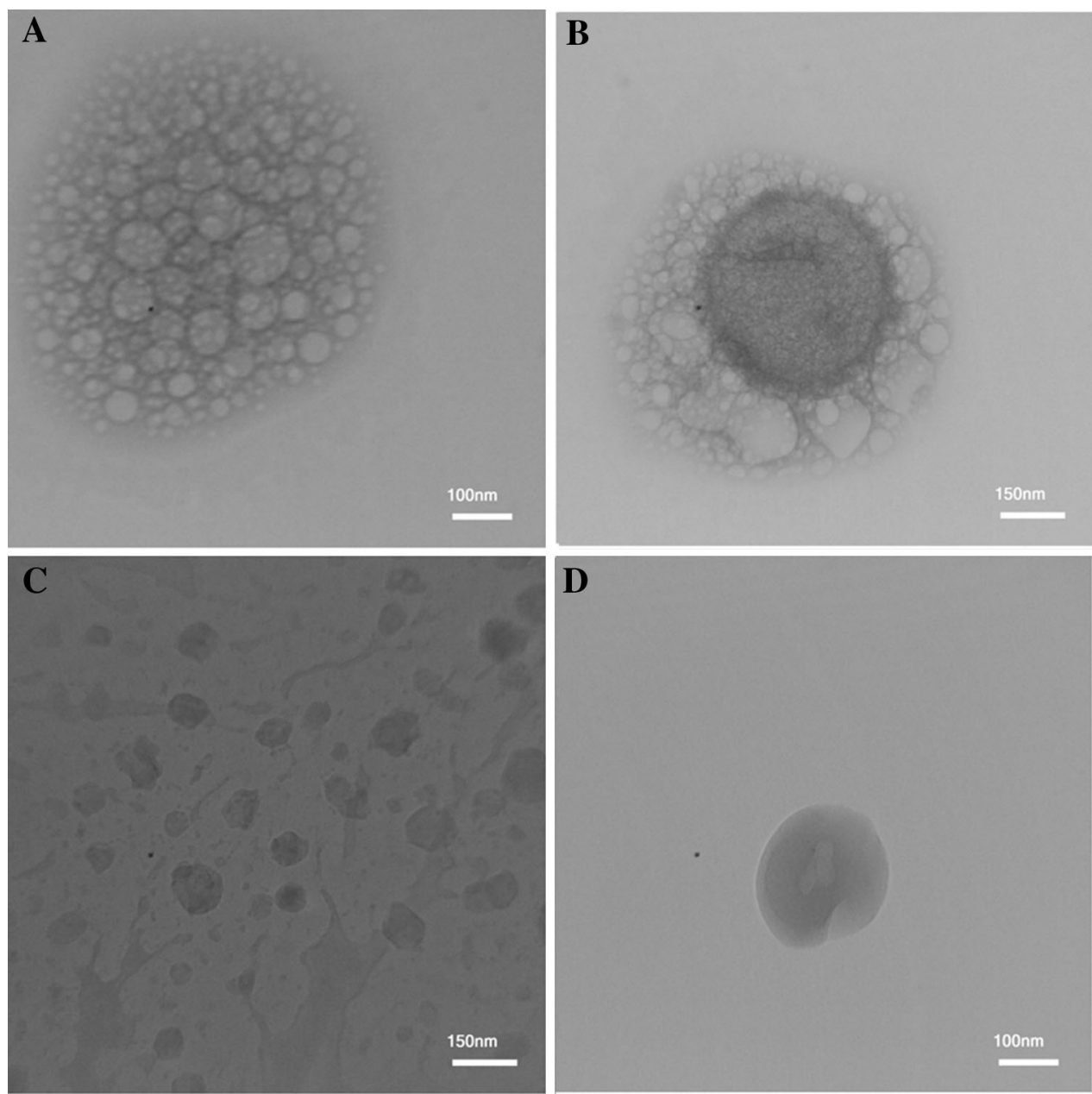


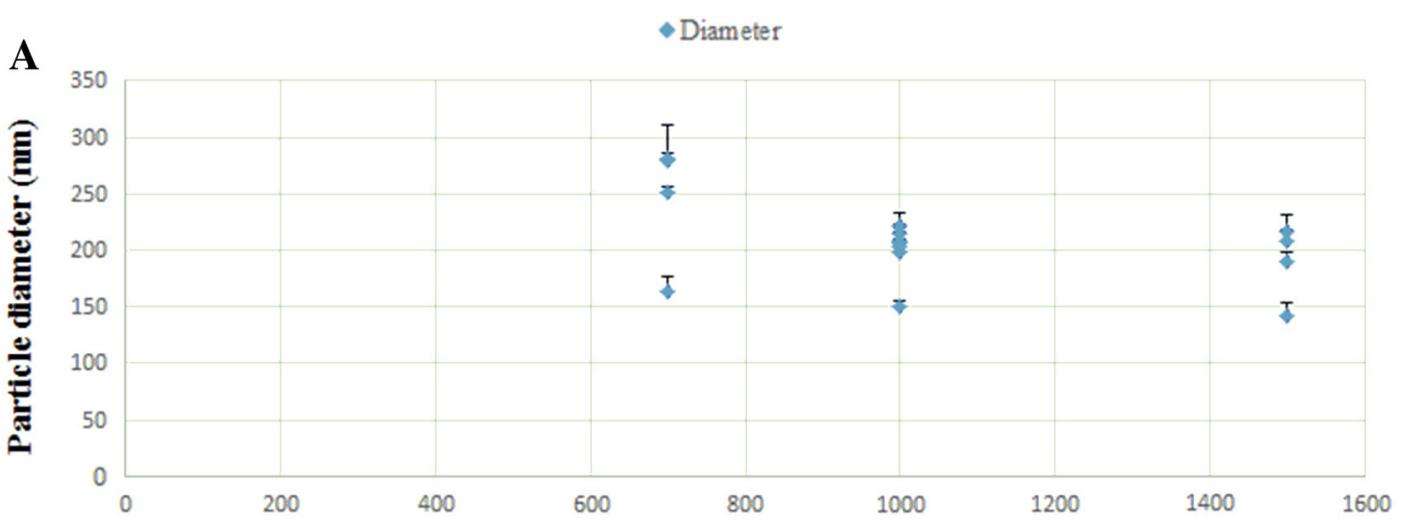

Pressure (bar)

B

- Diameter

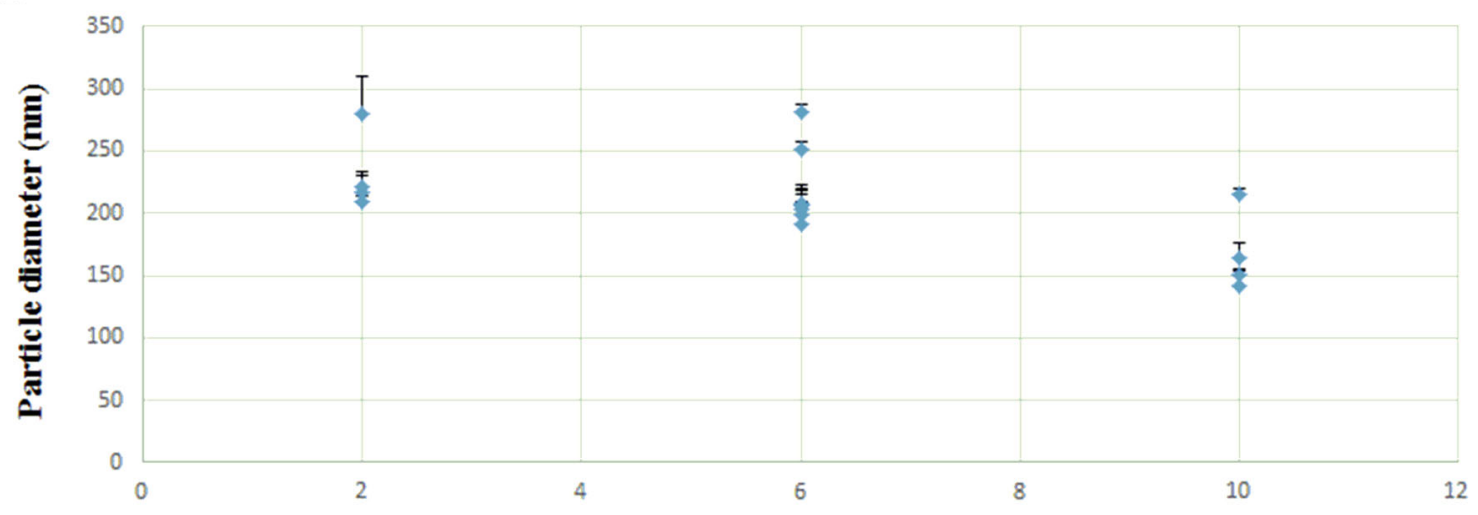

Time (min)

$\downarrow$ Diameter

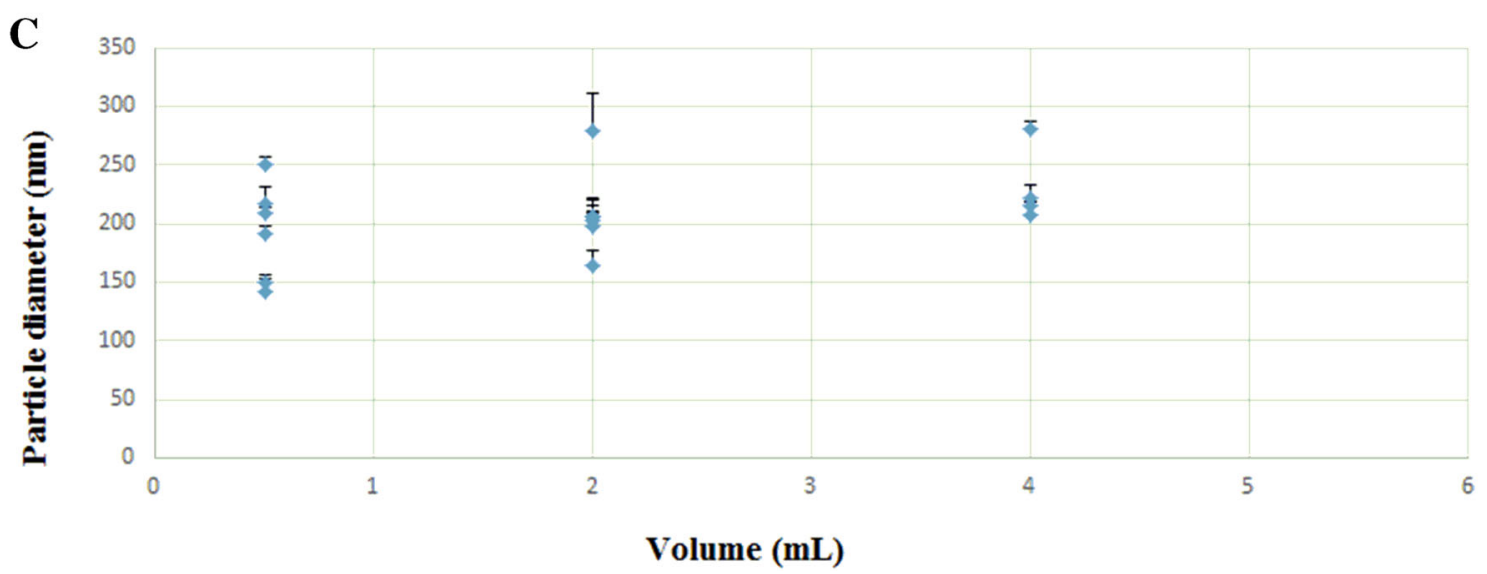

Fig. 5 Influence of the pressure value (a), time value (b), and volume value (c) on the particle diameter (nm) of HSA nanoparticles (mean \pm SD; $n=3$ ) 
Table 5 Intercept, coefficients, mean effects, and $p$ values for the predictive model obtained for three factors

\begin{tabular}{lrll}
\hline Variable & Coefficient & Mean effect $(\beta)$ & $p$ value \\
\hline Intercept & 802.817 & \multicolumn{1}{c}{-} & 0.000 \\
$\log P$ & -187.917 & -0.509 & 0.001 \\
$T^{2}$ & -0.820 & -0.663 & 0.000 \\
$V^{2}$ & 1.986 & 0.287 & 0.031 \\
\hline
\end{tabular}

$R^{2}=0.819$, standard error $=20.420$, and $F$-ratio $=19.550$

$P$ Pressure, $T$ Time, $V$ volume of organic solvent

shown with transmission electron microscopy

(TEM)

(CM30 300 kV, Berlin, Germany).

\section{Results and discussions}

The effect of three factors (under sonication and under $\mathrm{HPH}$ ) on the average diameter of HSA nanoparticles was evaluated for three times. The experiments were randomly performed for each factor on three levels using a BoxBehnken design, which provided enough information to calculate the regression model containing linear, interactions, and curved factor effects. The obtained values for the sonication and HPH experiments are presented in Tables 3 and 4 , respectively.
The mean diameter of the nanoparticles for all 17 experiments fit the polynomial model.

The model coefficients were calculated using a multiple regression technique and were validated using ANOVA with $p$ value $<0.05$. The criteria for the descriptive capability evaluation of a polynomial were the Fisher-ratio value $(F)$, adjusted $R^{2}$ and standard error of estimation (SE). The model, which successfully described the size and PdI of the nanoparticles, was the polynomial models including diverse numbers of terms and different combinations of factors.

Based on the independent sample $t$ test no significant difference between the DLS size of HSA nanoparticles under sonication and HPH was found $(p>0.05)$, resulting in acceptance of the null hypothesis. (The results of some experiments were shown in Fig. 2). The nanoparticles made using HPH were stable, whereas the nanoparticles made using sonication showed aggregation after 1 day. The SEM and TEM images in Figs. 3 and 4, respectively, confirm the results stated above.

The particle size in aforementioned pressure, time, and organic volume are summarized in Fig. 5. The volume, quadratic term of time, and logarithmic term of pressure significantly influenced the size of the nanoparticles. Therefore, the hyper-surface response in the multidimensional factor space is curved in the sphere of the experimental design. The existence of the logarithmic and second-order interactions between the principal factors in
Fig. 6 Predicted vs. experimental HSA nanoparticle size with regard 17 experimental conditions

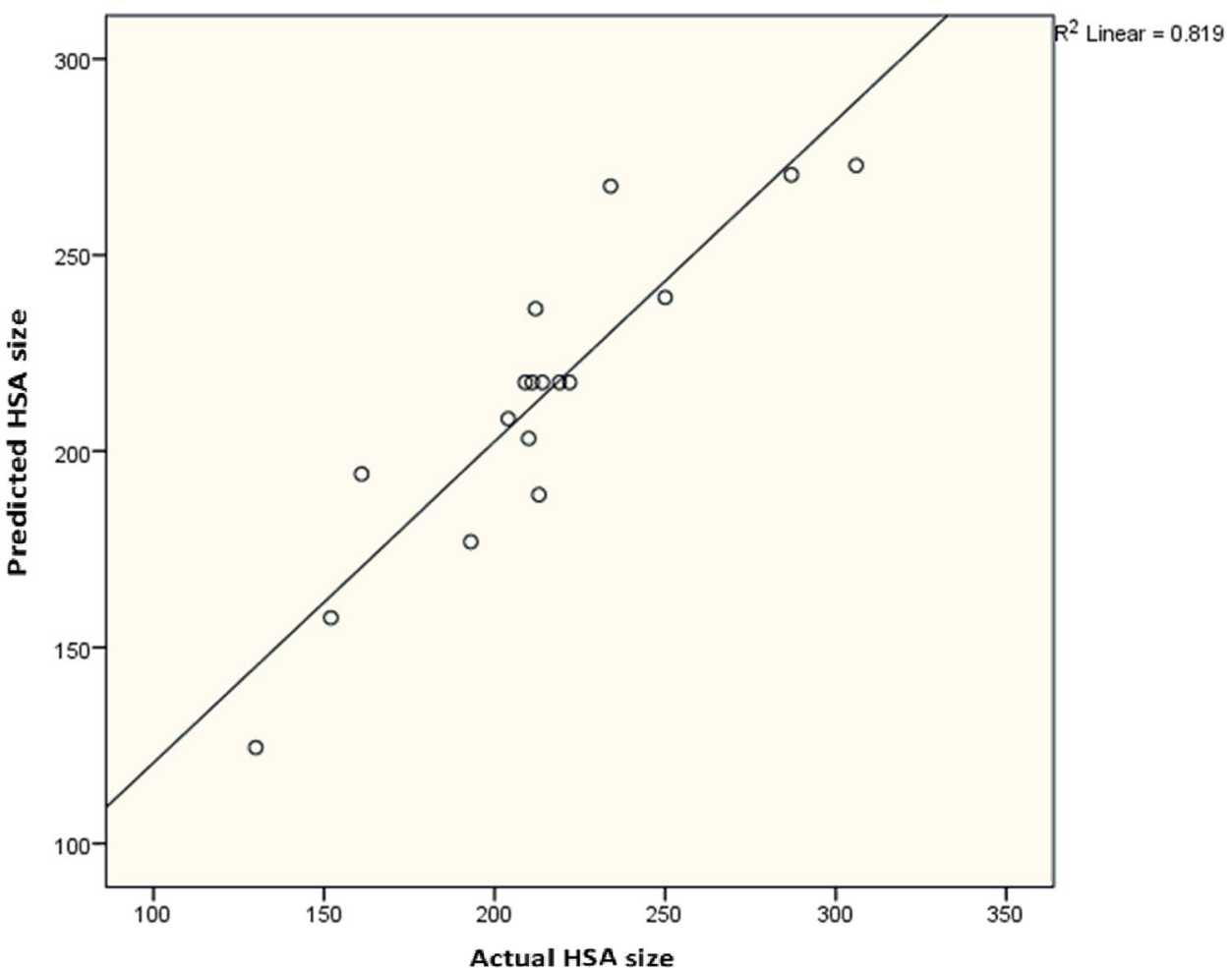


the conditions of the experiments emphasizes the need to conduct active multi-factor experiments to optimize of the nanoparticles preparation process. This indicates that the use of the multiple regression technique is essential in the optimization of the HSA nanoparticle preparation process. The size of the nanoparticles was predicted using the obtained equation:

$$
\begin{aligned}
& \left(-0.820 \times T^{2}\right)+\left(1.986 \times V^{2}\right)+(-187.917 \times \log P) \\
& +802.817
\end{aligned}
$$

The related model and its statistics are presented in Table 5, where $T$ is the time, $P$ is the pressure, and $V$ is the volume of organic solvent. The Standardized coefficient $(\beta)$ showed that the nanoparticles size follows the pressure and time as the most effective and the volume of organic solvent is minimally effective.

The null hypothesis was rejected based on the $p$ value, which is $<0.05$. Therefore, a relationship between the coefficients exists.

To assess the fitness of the obtained multiple linear regression (MLR) model, the residuals were analyzed statistically (Fig. 6). Figure 6 also shows a good correlation between the predicted and experimental results achieved under the optimized conditions. The fit of this model and the model predictability are quite satisfactory.

In the present study, we used acetonitrile instead of chloroform which was used in other reports for $n a b^{\mathrm{TM}}$ technology. Less toxicity of acetonitrile compared with chloroform [24] is advantage of this method. Although acetonitrile is a water-miscible solvent, the results showed that predictable particle size can be achieved for HSA nanoparticles.

\section{Conclusion}

The best conditions for the preparation of HSA nanoparticles was determined by a chemometric approach using a limited number of experiments. Moreover, the BoxBehnken design allows to determine a regression model that describes the dependence of particle size on the experimental parameters used in the HSA nanoparticle preparation. The results showed that particle size regularly changes as a function of pressure and time as the most effective factors. The existence of interactions between the factors in the conditions of the experiments emphasizes the need to use the multiple regression technique for the optimization of the HSA nanoparticle preparation process.

Acknowledgements This project was performed in Nanotechnology Research Centre, Tehran University of Medical Sciences (TUMS). The authors gratefully acknowledge Nano Daru pharmaceutical Company for the support to do this project. In addition, technical assistance of Navid Nateghian, Mehdi Esfandyari-manesh, Nazanin Shabani Ravari, and Faezeh Khosravi is appreciated.

Conflict of interest The authors declare that they have no conflict of interests.

Open Access This article is distributed under the terms of the Creative Commons Attribution 4.0 International License (http://creative commons.org/licenses/by/4.0/), which permits unrestricted use, distribution, and reproduction in any medium, provided you give appropriate credit to the original author(s) and the source, provide a link to the Creative Commons license, and indicate if changes were made.

\section{References}

1. Goodarzi, N., Ghahremani, M.H., Amini, M., Atyabi, F., Ostad, S.N., Shabani, N.R., Nateghian, N., Dinarvand, R.: CD44-targeted docetaxel conjugate for cancer cells and cancer stem-like cells: a novel hyaluronic acid-based drug delivery system. Chem. Biol. Drug Des. 83, 741-752 (2014)

2. Goodarzi, N., Varshochian, R., Kamalinia, G., Atyabi, F., Dinarvand, R.: A review of polysaccharide cytotoxic drug conjugates for cancer therapy. Carbohydr. Polym. 92, 1280-1293 (2013)

3. Saadat, E., Amini, M., Khoshayand, M.R., Dinarvand, R., Dorkoosh, F.A.: Synthesis and optimization of a novel polymeric micelle based on hyaluronic acid and phospholipids for delivery of paclitaxel, in vitro and in vivo evaluation. Int. J. Pharm. 475, 163-173 (2014)

4. Qiang, F., Sun, J., Zhang, W., Sui, X., Yan, Z., He, Z.: Nanoparticle albumin-bound (NAB) technology is a promising method for anti-cancer drug delivery. Recent Pat. Anticancer Drug Discov. 4, 262-272 (2009)

5. Nateghian, N., Goodarzi, N., Amini, M., Atyabi, F., Khorramizadeh, M.R., Dinarvand, R.: Biotin/folate-decorated human serum albumin nanoparticles of docetaxel: comparison of chemically conjugated nanostructures and physically loaded nanoparticles for targeting of breast cancer. Chem. Biol. Drug Des. 87, 1-14 (2015)

6. Esmaeili, F., Dinarvand, R., Ghahremani, M.H., Amini, M., Rouhani, H., Sepehri, N., Ostad, S.N., Atyabi, F.: Docetaxelalbumin conjugates: preparation, in vitro evaluation and biodistribution studies. J. Pharm. Sci. 98, 2718-2730 (2009)

7. Nasrollahi, Z., Mohammadi, S.R., Mollarazi, E., Yadegari, M.H., Mohammad Hassan, Z., Talaei, F., Dinarvand, R., Akbari, H., Atyabi, F.: Functionalized nanoscale $\beta$-1,3-glucan to improve Her2+ breast cancer therapy: in vitro and in vivo study. J. Control. Release 202, 49-56 (2015)

8. Taheri, A., Dinarvand, R., Nouri, F.S., Khorramizadeh, M.R., Borougeni, A.T., Mansoori, P., Atyabi, F.: Use of biotin targeted methotrexate-human serum albumin conjugated nanoparticles to enhance methotrexate antitumor efficacy. Int. J. Nanomed. 6, 1863-1874 (2011)

9. Zhen, J., Liang, X., Zhao, Y., Sun, Y., Hu, X., Fan, J.: Synthesis and characterization of folic acid-conjugated human serum albumin (HSA) nanoparticles for isoalantolactone cellular uptake in HeLa. Afr. J. Pharm. Pharmacol. 7, 1038-1045 (2013)

10. Elzoghby, A.O., Samy, W.M., Elgindy, N.A.: Albumin-based nanoparticles as potential controlled release drug delivery systems. J. Control. Release 157, 168-182 (2012)

11. Tavassolian, F., Kamalinia, G., Rouhani, H., Amini, M., Ostad, S.N., Khoshayand, M.R., Atyabi, F., Tehrani, M.R., Dinarvand, R.: Targeted poly(l- $\gamma$-glutamyl glutamine) nanoparticles of 
docetaxel against folate over-expressed breast cancer cells. Int. J. Pharm. 467, 123-138 (2014)

12. Moghimi, Farhangrazi: S.M., Shadi, Z.: just so stories: The random acts of anti-cancer nanomedicine performance. Nanomedicine 10, 1661-1666 (2014)

13. Goodarzi, N., Ghahremani, M.H.: Dinarvand: hyaluronic acidbased nano drug delivery systems against cancer stem cells. J. Med. Hypotheses Ideas 6, 65 (2012)

14. Wan, X., Zheng, X., Pang, X., Pang, Z., Zhao, J., Zhang, Z., Jiang, T., Xu, W., Zhang, Q., Jiang, X.: Lapatinib-loaded human serum albumin nanoparticles for the prevention and treatment of triple-negative breast cancer metastasis to the brain. Oncotarget 7, 34038-34051 (2016)

15. Danhier, F., Feron, O., Préat, V.: To exploit the tumor microenvironment: passive and active tumor targeting of nanocarriers for anti-cancer drug delivery. J. Control. Release 148, 135-146 (2010)

16. Singh, R., Sankar, C., Rajasree, P.H.: Human serum albumin nanoparticles for enhanced drug delivery to treat breast cancer: preparation and in vitro assessment. Intl $\mathbf{J}$ Pharm Life Sci. 3, 2055-2063 (2012)

17. Elsadek, B., Kratz, F.: Impact of albumin on drug delivery-new applications on the horizon. J. Control. Release 157, 4-28 (2012)

18. Narayanan, V., Weekes, C.D.: Nanoparticle albumin-bound (nab)-paclitaxel for the treatment of pancreas ductal adenocarcinoma. Gastrointest. Cancer Targets Ther. Dovepress 5, 11-19 (2015)
19. Desai, N.: Nab technology: a drug delivery platform utilizing endothelial gp60 receptor-based transport and tumour derived SPARC for targeting. Drug Delivery Report, 37-41 (2008)

20. Lomis, N., Westfall, S., Farahdel, L., Malhotra, M.: Human serum albumin nanoparticles for use in cancer drug delivery: process optimization and in vitro characterization. Nanomaterials (Basel) 6, 1-17 (2016)

21. Kratz, F.: Albumin as a drug carrier: design of prodrugs, drug conjugates and nanoparticles. J. Control. Release 132, 171-183 (2008)

22. Honary, S., Ebrahimi, P., Ghasemitabar, M.: Preparation of gold nanoparticles for biomedical applications using chemometric technique. Trop. J. Pharm. Res. 12, 295-298 (2013)

23. Honary, S., Ebrahimi, P., Asgari-rad, H., Mohamadpour, F.: Optimization of iron oxide nanoparticle preparation for biomedical applications by using Box-Behenken design. Int. J. Nanosci. Nanotechnol. 10, 257-261 (2014)

24. United States pharmacopoeia (USP 39-NF 34). Vol. 1, 339-354 (2016)

\section{Publisher's Note}

Springer Nature remains neutral with regard to jurisdictional claims in published maps and institutional affiliations. 